\title{
WEAK CONVERGENCE OF VEGTOR-VALUED SERIES AND INTEGRALS
}

\author{
R. E. EDWARDS
}

(received 14 May 1962)

\section{Introduction}

Throughout this paper $E, F$ and $G$ denote separated locally convex spaces, $F \subset G$, the injection $i: F \rightarrow G$ being continuous (i.e. the topology on $F$ is finer than that induced on it by the topology on $G) . E^{\prime}, F^{\prime}$ and $G^{\prime}$ denote the respective duals of $E, F$ and $G . i^{\prime}$ is the adjoint map of $G^{\prime}$ into $F^{\prime}$, which is defined by restricting linear forms on $G$ to $F \subset G$.

The "series case" of the type of problem we have in mind concerns the convergence of series of the type

$$
\sum_{k}\left\langle x, e_{k}^{\prime}\right\rangle g_{k},
$$

$\left(e_{k}^{\prime}\right)$ and $\left(g_{k}\right)$ being sequences of elements of $E^{\prime}$ and of $G$, respectively. Under certain conditions which we shall set out below, it happens that the convergence of (1) for each $x \in E$ and in the sense of the weakened topology $\sigma\left(G, G^{\prime}\right)$, each sum being in $F$, implies already the convergence or the weakened topology on $F$ (or, in some cases, even for the initial topology on $F$ ). If $E=F=G$ and the $e_{k}^{\prime}$ are the coefficient functionals associated with a Schauder base $\left(e_{k}\right)$ in $E$, results of this type are known to hold when $E$ is a Banach or a Fréchet space and spoken of as the "weak basis theorem".

It is only a short step to pass from series (1) to integrals of the type

$$
\bar{\int}_{T}\left\langle x, e_{t}^{\prime}\right\rangle g_{t} d \mu(t)
$$

$T$ being a locally compact space and $\mu$ a positive Radon measure on $T$.

In either case the summand or integrand, as the case may be, is a bilinear form on $E \times G^{\prime}$ which we may write as $\left\langle x\left|B_{t}\right| z^{\prime}\right\rangle . B: t \rightarrow B_{t}$ is thus analogous to a tensor field of mixed rank two over $T$. It is convenient to formulate the general problem in such terms. To do this we make the following conventions.

The field $B$ being given such that $t \rightarrow\left\langle x\left|B_{t}\right| z^{\prime}\right\rangle$ is essentially $\mu$-integrable for each pair $\left(x, z^{\prime}\right) \in E \times G^{\prime}$, we denote by $\bar{\int}\left\langle x\left|B_{t}\right| d \mu(t)\right.$ that element of $G^{\prime *}$ defined as

$$
z^{\prime} \rightarrow \bar{\int}\left\langle x\left|B_{\imath}\right| z^{\prime}\right\rangle d \mu(t)
$$


The integral $\left.\bar{\int}\left|B_{t}\right| z^{\prime}\right\rangle d \mu(t)$ is defined analogously. Naturally, $\bar{\int}\left\langle x\left|B_{t}\right| d \mu(t)\right.$ may or may not belong to $G$ when the latter is viewed as a subset of $G^{\prime *}$; and $\left.\bar{\int}\left|B_{t}\right| z^{\prime}\right\rangle d \mu(t)$ may or may not belong to $E^{\prime} \subset E^{*}$.

In all cases the first step is to establish the continuity of the pointwise limit $u$ of a sequence of maps $u_{n}$ of $E$ into $G$ defined by

$$
u_{n}(x)=\bar{\int} s_{n}(t)\left\langle x\left|B_{t}\right| d \mu(t),\right.
$$

the $s_{n}$ being summation factors. If this can be done, the convergence for the weakened topology on $F$ is derived from simple assumptions concerning the summability factors $s_{n}$.

With further specialisation of $E$, stronger results may be derived.

\section{Some hypotheses on the spaces $E, F$ and $G$}

It is convenient to have certain hypotheses concerning these vector spaces listed in a bunch for future reference.

[1.1] $E, F$ and $G$ are separated locally convex spaces such that $F \subset G$ and the injection $i: F \rightarrow G$ is continuous. The adjoint $i^{\prime}$ of $i$ is defined by $i^{\prime}\left(z^{\prime}\right)=z^{\prime} \mid F$, mapping $G^{\prime}$ into $F^{\prime}$. Note that $i^{\prime}\left(G^{\prime}\right)$ is total (= weakly dense) in $F^{\prime}$, i.e. it separates points of $F$.

[1.2] $u_{n}(n \in N$, the set of natural numbers) are linear maps of $E$ into $G$ such that $z^{\prime} \circ u_{n}$ is continuous on $E$ for each pair $\left(z^{\prime}, n\right) \in G^{\prime} \times N$.

[1.3] For each $x \in E, u(x)=\lim _{n} u_{n}(x)$ exists in the sense of the topology $\sigma\left(G^{\prime}, G\right)$, and $u(x) \in F$.

[1.4] There exists a base $\{W\}$ of weak neighbourhoods of 0 in $F$, each of which is closed in $G$.

[1.5] There exists a base $\{V\}$ of neighbourhoods of 0 in $F$, each of which is such that $i^{\prime}\left(G^{\prime}\right) \cap V^{0}$ is weakly dense in $V^{0}\left(V^{0}\right.$ being the polar in $F^{\prime}$ of $V(F)$.

Note that if $E$ is barrelled, or bornological, or relatively strong, or if the closed graph theorem is true for linear maps of $E$ into $G$ (a situation which we symbolise: $(E, G) \in(\mathrm{cgt}))$, then [1.2] is true if and only if $u_{n}$ is continuous on $E$ into $G$.

If $[1.5]$ is true, there is a base $\{V\}$ of neighbourhoods of 0 in $F$, each of which is closed, convex and balanced and which moreover satisfies the condition set forth in the statement [1.5].

\section{The continuity of $u$ on $E$ into $F$}

We shall give two sufficient sets of conditions in order that $u$ be continuous on $E$ into $F$. 
THEOREM A. Suppose that $E$ is barrelled and that [1.1], [1.2], [1.3] and $[1.5]$ are satisfied. Then $u$ is continuous on $E$ into $F$.

Proof. Taking any closed, convex and balanced neighbourhood $V$ of 0 in $F$, chosen as in [1.5], it is enough to show that $q \circ u$ is continuous on $E$, $q$ being the gauge of $V$ (i.e. a seminorm on $F$ such that $V$ is precisely the set of $y \in F$ satisfying $q(y) \leqq 1)$. Now

$$
q(u(x))=\operatorname{Sup}\left\{\left|\left\langle u(x), y^{\prime}\right\rangle\right|: y^{\prime} \in V^{0}\right\},
$$

$V^{0}$ being the polar in $F^{\prime}$ of $V$. According to [1.5] this equals

$$
\operatorname{Sup}\left\{\left|\left\langle u(x), y^{\prime}\right\rangle\right|: y^{\prime} \in V^{0} i^{\prime}\left(G^{\prime}\right)\right\} \text {. }
$$

Since $E$ is barrelled, it suffices to show that $y^{\prime} \circ u$ is continuous on $E$ for each $y^{\prime} \in i^{\prime}\left(G^{\prime}\right)$. But if $y^{\prime}=i^{\prime}\left(z^{\prime}\right)$ for some $z^{\prime} \in G^{\prime},[1.3]$ shows that

$$
\begin{aligned}
\left\langle u(x), y^{\prime}\right\rangle & =\left\langle u(x), i^{\prime}\left(z^{\prime}\right)\right\rangle=\left\langle u(x), z^{\prime}\right\rangle \\
& =\lim _{n}\left\langle u_{n}(x), z^{\prime}\right\rangle .
\end{aligned}
$$

By [1.2], each $z^{\prime} \circ u_{n}$ is continuous on $E$. So, again since $E$ is barrelled, one concludes that $y^{\prime} \circ u$ is continuous on $E$ and the proof is complete.

THEOREM B. Suppose that $[1.1]-[1.3]$ hold, that $u_{n}(E) \subset F$ for each $n$, and that $(E, F) \in(\mathrm{cgt})$. Then each $u_{n}$ is continuous on $E$ into $F$. If also $E$ is barrelled, then $u$ is continuous on $E$ into $F$.

Proof. If $u_{n}(E) \subset F,[1.2]$ shows that $u_{n}$ has a graph closed in $E \times F$. Hence $u_{n}$ is continuous on $E$ into $F$.

To show that $u$ is continuous on $E$ into $F$, it suffices to show that its graph also is closed in $E \times F$. Thanks to [1.1], this will follow if one shows that $z^{\prime} \circ u$ is continuous on $E$ for each $z^{\prime} \in G^{\prime}$. But $z^{\prime} \circ u$ is the pointwise limit of the $z^{\prime} \circ u_{n}$, as a consequence of [1.3]. Since $E$ is barrelled, it follows that $z^{\prime} \circ u$ is continuous.

Notes. (1) If in Theorem $\mathrm{A}$ we are given also that $u_{n}(E) \subset F$, then (as in the proof of Theorem B) one may infer that each $u_{n}$ is continuous on $E$ into $F$.

(2) In connection with Theorem B it is perhaps useful to recall cases in which one can be certain that $(E, F) \in(\mathrm{cgt})$. This is indeed the case provided one of the following conditions is satisfied.

(a) $E$ is an inductive limit, and $F$ a countable inductive limit, of Fréchet spaces, moreover in this case one may replace "closed graph" by "sequentially closed graph";

(b) $E$ is barrelled and $F$ is $B_{r}$-complete. 


\section{Hypotheses concerning the field $B$}

As before it is convenient to group together a number of hypotheses.

[3.1] $\left(s_{n}\right)$ is a sequence of scalar-valued functions on $T$ such that for each $m$ boundedly.

$$
\lim _{n} s_{n} s_{m}=s_{m}
$$

[3.2] For each $x \in E$ there exists a weak Cauchy sequence $\left(x_{n}^{*}\right)$ in $E$ such that for each $n \in N$ (the set of natural numbers) and $z^{\prime} \in G^{\prime}$

$$
s_{n}(t)\left\langle x \mid B(t) / z^{\prime}\right\rangle=\left\langle x_{n}^{*}|B(t)| z^{\prime}\right\rangle
$$

for locally almost all $t \in T$.

[3.3] For each triplet $\left(x, z^{\prime}, n\right) \in E \times G^{\prime} \times N$ the function $s_{n}\left\langle x B z^{\prime}\right\rangle$ is essentially integrable and the linear form

$$
z^{\prime} \rightarrow \bar{\int} s_{n}\left\langle x|B| z^{\prime}\right\rangle d \mu
$$

is weakly continuous on $G^{\prime}$, so that

$$
u_{n}(x)=\bar{\int} s_{n}\langle x|B| d \mu
$$

exists as an element $x$ of $G$.

[3.4] For each pair $\left(n, z^{\prime}\right) \in N \times G^{\prime}$ the linear form

$$
x \rightarrow \bar{\int} s_{n}\left\langle x|B| z^{\prime}\right\rangle d \mu
$$

is continuous on $E$, so that

$$
\left.v_{n}\left(z^{\prime}\right)=\bar{\int} s_{n}|B| z^{\prime}\right\rangle d \mu
$$

exists as an element of $E^{\prime}$. ( $v_{n}$ is the adjoint of $u_{n}$ qua map of $E$ into $G$ ).

[3.5] For each $x \in E$ the limit

$$
u(x)=\lim u_{n}(x)
$$

exists in the sense of the weakened topology $\sigma\left(G, G^{\prime}\right)$, the limit $u(x)$ belonging to $F(C G)$.

\section{Cases in which convergence of $\left(u_{n}(x)\right)$ for $\sigma\left(G, \sigma^{\prime}\right)$ implies that for $\sigma\left(F, F^{\prime}\right)$}

Consider any case in which $u$ is known to be continuous on $E$ into $F$. Then $u$ will transform weak Cauchy (resp. weakly convergent, convergent) sequences in $E$ into weak Cauchy (resp. weakly convergent, convergent) sequences in $F$. In particular, if [3.2] holds, the sequence $\left(u\left(x_{m}^{*}\right)\right)=\left(y_{m}\right)$, say, is weakly Cauchy in $F$. On the other hand, one has by definition

$$
y_{m}=\lim _{n} u_{n}\left(x_{m}^{*}\right)
$$


weakly in $G$, so that for each $z^{\prime} \in G^{\prime}$ one has

$$
\begin{aligned}
\left\langle y_{m}-u\left(x_{m}\right), z^{\prime}\right\rangle & =\lim _{m} \bar{\int} s_{n}\left\langle x_{m}^{*}|B| z^{\prime}\right\rangle d \mu-\bar{\int} s_{m}\left\langle x|B| z^{\prime}\right\rangle d \mu \\
& =\lim _{m} \int\left(s_{n}-1\right) s_{m}\left\langle x|B| z^{\prime}\right\rangle d \mu,
\end{aligned}
$$

which, thanks to [3.1] and [3.3], is zero. Accordingly, $y_{m}=u_{m}(x)$ and so, by what has already been said, $\left(u_{m}(x)\right)$ is Cauchy for $\sigma\left(F, F^{\prime}\right)$. If [1.4] holds one may infer that $\left(u\left(x_{m}\right)\right)$, being weakly convergent in $G$ to $u(x)$ and weakly Cauchy in $F$, must be weakly convergent in $F$ to $u(x)$.

This permits us to formulate a third theorem.

THEOREM C. Suppose satisfied either of the following two sets of hypotheses

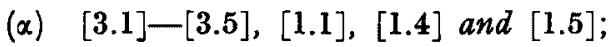

(B) $[3.1]-[3.5],[1.4]$, and $(E, F) \in(\mathrm{cgt})$.

Then, if $E$ is barrelled, the sequence $\left(u_{m}(x)\right)$ is convergent to $u(x)$ for the topology $\sigma\left(F, F^{\prime}\right)$.

If it is known that $\left(x_{m}^{*}\right)$ is convergent (resp. weakly convergent) in $E$ to $x$, then [1.5] may be dropped and one may conclude that $u_{m}(x) \rightarrow u(x)$ in the sense of the initial topology (resp. the weakened topology) on $F$.

\section{Specialisation of $E$ and $F$}

At the expense of specialising $E$ and (to a lesser extent) $F$, one can strengthen the conclusions of Theorem $\mathrm{C}$. The additional properties concerning $E$ are those introduced by Grothendieck [1] and called the strict DunfordPettis property (SDPP for short) and the Dieudonné property (DP for short).

Let us add to our hypotheses on $E$ and $F$ the following

[1.6] $E$ possesses both the SDPP and the DP;

[1.7] $F$ is complete and weakly sequentially complete. If $u$ is continuous on $E$ into $F,[1.7]$ shows that $u$ transforms weak Cauchy sequences in $E$ into weakly convergent sequences in $F$ (and in any case transforms weak Cauchy sequences in $E$ into weak Cauchy sequences in $F$ ). Assuming [1.6] we may infer that

(i) $u$ transforms bounded sets in $E$ into weakly relatively compact sets in $F$,

this since $E$ has the DP. Since also $E$ has the SDPP it follows that

(ii) $u$ transforms weak Cauchy sequences in $E$ into convergent sequences in $F$;

(iii) $u$ transforms weakly relatively compact sets in $E$ into relatively compact sets in $F$; 
(iv) $u$ transforms bounded and weakly metrisable sets in $E$ into relatively compact sets in $F$.

In particular one arrives at the following theorem.

THEOREM D. Suppose satisfied either of the two sets of hypotheses $(\alpha)$ and $(\beta)$ of Theorem $C$, and also [1.6] and [1.7]. Then $\left(u_{n}(x)\right)$ converges in $F$ to $u(x)$ for each $x \in E$.

Note. Clearly, if [3.2] holds with "weak Cauchy" replaced by "weakly convergent" or "convergent", one may neglect $[1.6]$ and $[1.7]$ in deducing that $\left(u\left(x_{n}\right)\right)$ is weakly convergent or convergent, as the case may be.

Notable cases in which [1.6] and [1.7] are satisfied are those in which $E$ is a space $C_{0}(S)$ (continuous functions on a locally compact space $S$ which vanish at infinity), or $C^{r}(O), O$ being an open subset of $R^{m}$, or if $E$ is boundedly compact (in which case [1.7] is superfluous), and if $F$ is a space $L^{1}$. See [1], pp. 139-152, 161 .

Moreover, if $E$ is a space $L^{\infty}$, it is isomorphic with a space $C_{0}(S)$ in which $S$ is a compact Stonian space. If $F$ is complete and separable, any continuous linear $u$ mapping $E$ into $F$ transforms weak Cauchy sequences into convergent sequences. Thus, with the hypotheses in Theorem $C, u$ will transform $\left(x_{n}\right)$ into a sequence converging to $u(x)$. See [1], pp. 168, 137.

\section{Some examples}

The "integral case" is at least as significant as the "series case". However, although there are no new essential difficulties in the former case, it is inevitably more complicated in detail. For this reason we restrict the illustrations to the case of series.

(1) The "series case" is always reducible to the situation in which $T$ is the set $N$ of natural numbers endowed with the discrete topology and $\mu$ is the measure placing a unit mass at each point of $N$. Then $\int_{T} \cdots d \mu=\sum_{N}$ $(\cdots)$, the series being absolutely convergent. The simplest, though not the only, choice of $s_{n}$ is as the characteristic function of the segment $[1, n]$ of $N$, whilst $\left\langle x B(n) z^{\prime}\right\rangle=x(n) \cdot\left\langle g_{n}, z^{\prime}\right\rangle$. This applies if, as we shall suppose, $E$ is a space of sequences and $\left(g_{n}\right)$ is a sequence of elements of $G$. Then [3.1] is satisfied in the stronger form $s_{n} s_{m}=s_{m}$ if $n>m$ and $0 \leqq s_{n} \leqq 1$ for all $n$. Condition [3.2] will be satisfied if $E$ contains each finite section $x_{n}^{*}$ of $x$ whenever $x \in E$,

$$
x_{n}^{*}(k)=\left\{\begin{array}{lll}
x(n) & \text { if } & k \leqq n \\
0 & \text { if } & x>n
\end{array}\right.
$$

[3.3] is visibly satisfied and

$$
u_{n}(x)=\sum_{1 \leqq k \leq n} x(k) g_{k}
$$


[3.4] requires merely that each coordinate function $x \rightarrow x(k)$ is continuous on $E$. Finally $[3.5]$ is the major premise and demands that the series $\sum_{k} x(k) g_{k}$ is weakly convergent in $G$ to a sum which belongs to $F$.

Even at this stage there remains considerable freedom in the choice of $E$ and of $F$. If this is done with respect for $[1.1]-[1.5]$, the conclusion will be that the series (*) is weakly convergent in $F$.

To take a specific case, suppose that $\Omega$ is a domain in $R^{r}$ and take for $G$ the space $D^{\prime}(\Omega)$ of Schwartz distributions on $\Omega$. For $F$ we may take any of the spaces $L^{p}(\Omega), L_{\text {loc }}^{p}(\Omega), C^{q}(\Omega)$ or $D^{q}(\Omega)$ where $1 \leqq p<\infty$ and $q$ is either a natural number of $\infty$. In each case $i^{\prime}\left(G^{\prime}\right)$ is $D(\Omega)$, the space of test functions. It is easily verified that both [1.4] and [1.5] are satisfied. Moreover, save perhaps in the case where $F=D^{a}(\Omega)$, the closed graph theorem is available for linear maps of $E$ into $F$, provided $E$ is barrelled; and if $F=D^{q}(\Omega)$, it is available whenever $E$ is an inductive limit of Fréchet spaces.

The conclusion would be that the series (*), if convergent for each $x \in E$ in the sense of distributions to a sum which lies in $F$, then the series converges weakly in $F$. With further restrictions on $E$ this conclusion may be strenthened as indicated in $\S 5$.

(2) This example is concerned with biorthogonal expansions.

Suppose that $\left(e_{k}\right)$ and $\left(e_{k}^{\prime}\right)$ are biorthogonal sequences in $E$ and $E^{\prime}$, so that

$$
\left\langle e_{i}, e_{j}^{\prime}\right\rangle=\delta_{i j} \text {. }
$$

Suppose also that $\left(f_{k}\right)$ is a sequence in $F$.

We introduce summation factors $\alpha_{n}(k)$ and put

$$
u_{n}(x)=\sum_{k} \alpha_{n}(k)\left\langle x, e_{k}^{\prime}\right\rangle f_{k},
$$

and we assume that for each $x$ in $E$

$$
u(x)=\lim _{n} u_{n}(x)
$$

exists weakly in $G$ and belongs to $F$.

The usual concept of biorthogonal expansions is generalised to the extent of admitting summation factors $\beta_{m}(k)$ and assuming that for certain (not necessarily all) $x \in E$ one has

$$
x=\lim _{m} s_{m}=\lim _{m} \sum_{k} \beta_{m}(k)\left\langle x, e_{k}^{\prime}\right\rangle e_{k},
$$

the limit existing in the sense of the weakened (resp. initial) topology on $E$.

If $u$ is continuous on $E$ into $F$ (see Theorems $\mathrm{A}$ and $\mathrm{B}$ ), it will follow that $u\left(s_{m}\right) \rightarrow u(x)$ for the weakened (resp. initial) topology on $F$.

On the other hand, if

$$
\sum_{k}\left|\beta_{m}(k)\left\langle x, e_{k}^{\prime}\right\rangle\left\langle f_{k}, z^{\prime}\right\rangle\right|<+\infty
$$


for each $z^{\prime} \in G^{\prime}$ and each $m$, and if

and

$$
\lim _{n} \alpha_{n}(k) \beta_{m}(k)=\beta_{m}(k) \quad(m, k=1,2, \cdots)
$$

$$
\left|\alpha_{n}(k) \beta_{m}(k)\right| \leqq c_{m}\left|\beta_{m}(k)\right| \quad(n, k=1,2, \cdots),
$$

it will follow that

$$
u\left(s_{m}\right)=\sum_{k} \beta_{k}(k)\left\langle x, e_{k}^{\prime}\right\rangle f_{k} .
$$

One concludes therefore that

$$
\lim _{m} \sum_{k} \beta_{m}(k)\left\langle x, e_{k}^{\prime}\right\rangle f_{k}=u(x)
$$

in the sense of the weakened (resp., initial) topology on $F$, whenever $x \in E$ and (**) holds.

When $E=F=G$ are Fréchet spaces, and

$$
\alpha_{n}(k)=\beta_{n}(k)=\left\{\begin{array}{lll}
1 & \text { for } k \leqq n \\
0 & \text { for } k>n,
\end{array}\right.
$$

results of this type are analogous to Banach's "weak basis theorem".

\section{Reference}

[1] A. Grothendieck, Sur les applications faiblement compactes d'espaces du type $C(K)$, Canadian Journ. Math. 5 (1953), 129-173.

Department of Mathematics,

Inst. of Advanced Studies, A.N.U.,

Canberra. 